\title{
EFFICIENT HEDGING WHEN ASSET PRICES FOLLOW A GEOMETRIC POISSON PROCESS WITH UNKNOWN INTENSITIES*
}

\author{
MICHAEL KIRCH ${ }^{\dagger}$ AND WOLFGANG J. RUNGGALDIER R $^{\ddagger}$
}

\begin{abstract}
We consider the problem of determining a strategy that is efficient in the sense that it minimizes the expectation of a convex loss function of the hedging error for the case when prices change at discrete random points in time according to a geometric Poisson process. The intensities of the jump process need not be fully known by the investor. The solution algorithm is based on dynamic programming for piecewise deterministic control problems, and its implementation is discussed as well.
\end{abstract}

Key words. geometric Poisson process, piecewise deterministic control problems, incomplete information, incomplete markets, efficient hedging, Bayesian approach

AMS subject classifications. Primary, 91B28, 93E20; Secondary, 91B70, 90C39, 60G55

DOI. $10.1137 /$ S0363012903423168

1. Introduction. This paper concerns the problem of hedging a future liability. Depending on the hedging criterion, various approaches have been considered in the literature. A mathematically attractive criterion, related to mean-variance hedging, is the quadratic criterion. This criterion leads to the mathematical problem of approximating an $L^{2}$-random variable by stochastic integrals. Starting from the work by Föllmer and Sondermann [9] and Schweizer [22], much research has been dedicated to this criterion so that this topic can by now be considered as a well-studied one (for a survey with extensive literature see [24]). For more general criteria the approaches are in a sense in common with utility maximization. They are mainly the so-called martingale approach (see, e.g., [3], [16], [13], [21]) and approaches based on optimal stochastic control.

One of the main goals in the present paper is to study the hedging problem in the context of incomplete/partial information on the underlying price evolution model. The various approaches mentioned above have, to some extent, also been applied to the case of incomplete information. For the quadratic/mean variance approach and under a martingale measure see, e.g., [23] and [10]. The martingale approach for the case of incomplete information was first considered in [18]. For more recent studies see, e.g., [4], [14], [26]; they concern mainly diffusion-type models where the uncertainty is in the stock appreciation rates that are supposed to be unknown constants and treated, from the Bayesian point of view, as random variables with a given prior distribution. For a more typically stochastic control approach see [20]. In the present paper we concentrate on the stochastic control approach that can indeed be viewed as a rather general approach for problems with partial information.

Stochastic control methods, in particular the method of dynamic programming (DP), have been applied mainly to diffusion-type models, and here DP leads to HJB-

*Received by the editors February 24, 2003; accepted for publication (in revised form) March 2, 2004; published electronically December 1, 2004.

http://www.siam.org/journals/sicon/43-4/42316.html

${ }^{\dagger}$ Department of Financial and Actuarial Mathematics, 8-10 Wiedner Hauptstrasse, 1040 Vienna, Austria (michael.kirch@gmx.net). Current address: Goldman Sachs, Peterborough Court, 133 Fleet Street, London EC4A 2BB (michael.kirch@gs.com). The research of this author was partially supported by the EU RTM-project Dynstoch (IMP, Fifth Framework Programme).

${ }_{\ddagger}$ Dipartimento di Matematica Pura ed Applicata, Universitá di Padova, 7 Via Belzoni, 35131 Padova, Italy (runggal@math.unipd.it). 
type equations; much current research is going on concerning analytical solutions to these equations. Diffusion-type models lead to continuous trajectories for the prices. In reality, prices change at discrete random points in time, and, in addition, they move at fixed increments (multiples of tick size). In this paper we consider a price evolution model that possesses these features, namely a geometric Poisson process (see also [15]). In order to concentrate better on the main issues, we consider a rather simple such model with a single risky asset and assume that the liability to be hedged is adapted to the filtration generated by the underlying price evolution. The approach can, however, be extended to markets with many assets, not all of them available for hedging, and with a liability that may be adapted to the filtration generated by all these assets. Furthermore, the simple geometric Poisson process can be extended to include compound Poisson processes. This simple model is also a natural generalization of the successful binomial (or Cox-Ross-Rubinstein) model, in which prices change at fixed proportions either up or down. While in the binomial model the changes occur at fixed points in time; here they occur more realistically at random points in time. In any case, an important test for a model is to check whether it is able to reproduce option prices observed in the market. The simple model (1) passes this test surprisingly well: it is able to reproduce both the smile observed in a foreign exchange market and the skew observed in equity markets.

A geometric Poisson process is driven by random jump processes that are characterized by their intensities. We assume the intensities to be constant in time, but, since one of our purposes is to highlight the problem of model uncertainty, we allow for the possibility that these intensities are not fully known to the investor. Taking the Bayesian point of view, they are considered as random variables with a distribution that is continuously updated on the basis of the information coming from observing the actual price evolution. This allows us to capture some of the most important features while keeping complexity low.

In the setting as described above, the market is incomplete, and so it is not possible to obtain for any given claim a self-financing and perfect hedging strategy. As a hedging criterion we consider here the expected value of a convex loss function applied to the hedging error and call a hedging strategy that minimizes this criterion efficient (or optimal). Notice that this includes the quadratic criterion and also the well-known shortfall risk criterion (see, e.g., [2], [8]).

For our geometric Poisson models, the DP approach of stochastic control leads to Bellman equations for piecewise deterministic processes (see, e.g., [5], [25], [6], [1]) that are studied here both under full as well as partial information on the underlying price evolution model.

In summary, we consider the following optimization problem where, letting all the prices be discounted with respect to the nonrisky asset, $X_{t}$ denotes the (for simplicity scalar) price process, $V_{t}$ is the value process corresponding to a self-financing and predictable investment strategy $\xi_{t}$, and $F\left(X_{S}\right)$ is the claim for a fixed maturity $S$. The processes $N_{t}^{+}$and $N_{t}^{-}$are jump processes where the intensity need not be fully known, $a, b$ are given positive constants, and $l(\cdot)$ is an increasing and convex loss function:

$$
\left\{\begin{array}{l}
d X_{t}=X_{t-}\left[\left(e^{a}-1\right) d N_{t}^{+}+\left(e^{-b}-1\right) d N_{t}^{-}\right] \\
d V_{t}=\xi_{t} X_{t-}\left[\left(e^{a}-1\right) d N_{t}^{+}+\left(e^{-b}-1\right) d N_{t}^{-}\right] \\
E\left\{l\left(F\left(X_{S}\right)-V_{S}\right)\right\} \quad \longrightarrow \quad \min .
\end{array}\right.
$$


The outline of the paper is as follows. In section 2 we describe more precisely the problem setup and obtain some preliminary results. The DP approach for piecewise deterministic processes is studied, in the context of our problem, in section 3 and is extended to the case of incomplete information in section 4, more precisely in subsection 4.2. Since, in the context of our model, the incomplete information concerns uncertainty about the intensities of the driving Poisson processes, in subsection 4.1 we recall some facts about the Bayesian approach to uncertain intensities. The computation of real-size problems needs some approximations to be introduced. These are discussed in section 5, where also an example is given to better illustrate the approximation procedure itself.

2. Problem setup. We examine efficient strategies in the situation where, considering for simplicity only a single risky asset, its price follows a geometric Poisson process, i.e.,

$$
X_{t}=x_{0} e^{a N_{t}^{+}-b N_{t}^{-}}
$$

for two independent Poisson processes $N^{+}, N^{-}$with intensities $\lambda^{+}, \lambda^{-}$defined on a filtered probability space $\left(\Omega, \mathcal{F}, \mathcal{F}_{t}, P\right)$ and constants $a, b>0$. To keep the presentation as simple as possible, we shall assume that all processes/values are already discounted; i.e. we implicitly assume the short rate $r_{t}$ to be equal to zero. The more realistic case of $r_{t}>0$ would not change the essence of the results but would considerably complicate the presentation.

For a hedging strategy $\xi$, where $\xi_{t}$ denotes the number of units of the risky asset held in the portfolio at time $t$, and an initial capital $V_{0}$, we define the associated wealth process by

$$
V_{t}=V_{0}+\int_{0}^{t} \xi_{s} d X_{s}
$$

thereby enforcing $\xi$ to be self-financing. Although we shall not pursue this here, transaction costs may be easily incorporated by subtracting from the right-hand side in (2) the total amount of transaction costs incurred up to time $t$. In order to keep the results below as general as possible, we shall consider $\xi_{t}$ to be real valued. We say that a strategy $\xi$ is admissible for initial capital $V_{0}$ if it is predictable and the associated wealth process satisfies

$$
V_{t} \geq-c, \quad t \in[0, S], \quad P \text {-a.s. }
$$

for some fixed $c \geq 0$ and a given time horizon $S$. Let $\mathcal{A}_{V_{0}}$ denote the class of all admissible strategies for initial capital $V_{0} \geq-c$.

We denote by $\tau_{n}$ the time of the $n$th jump

$$
\begin{aligned}
& \tau_{n}=\inf \left\{t \geq 0 \mid N_{t}^{+}+N_{t}^{-}=n\right\}, \\
& \hat{\tau}_{n}:=\tau_{n} \wedge S .
\end{aligned}
$$

Proposition 2.1. We have $\xi \in \mathcal{A}_{V_{0}}$ if and only if $\xi$ is predictable and satisfies

$$
\xi_{t} \in\left[-\frac{c+V_{\hat{\tau}_{n}}}{X_{\hat{\tau}_{n}}\left(e^{a}-1\right)}, \frac{c+V_{\hat{\tau}_{n}}}{X_{\hat{\tau}_{n}}\left(1-e^{-b}\right)}\right], \quad t \in\left(\hat{\tau}_{n}, \hat{\tau}_{n+1}\right], \quad n=0,1, \ldots, \quad P \text {-a.s. }
$$

Proof. First, observe that

$$
(0, S]=\bigcup_{n=0,1, \ldots}\left(\hat{\tau}_{n}, \hat{\tau}_{n+1}\right], \quad P \text {-a.s. }
$$


holds; i.e., condition (3) is satisfied if and only if it is satisfied on every interval $\left(\hat{\tau}_{n}, \hat{\tau}_{n+1}\right]$.

Since $X$ is a pure jump process, we obtain from (2)

$$
\begin{aligned}
& V_{t}=V_{\hat{\tau}_{n}}+\xi_{t}\left(X_{\hat{\tau}_{n+1} \wedge t}-X_{\hat{\tau}_{n}}\right), \quad t \in\left(\hat{\tau}_{n}, \hat{\tau}_{n+1}\right] \\
& = \begin{cases}V_{\hat{\tau}_{n}} & \text { for } t \in\left(\hat{\tau}_{n}, \hat{\tau}_{n+1}\right), \\
V_{\hat{\tau}_{n}}+\xi_{t} X_{\hat{\tau}_{n}}\left(e^{a}-1\right) & \text { for } t=\hat{\tau}_{n+1}, N_{t}^{+}-N_{t-}^{+}=1, \\
V_{\hat{\tau}_{n}}+\xi_{t} X_{\hat{\tau}_{n}}\left(e^{-b}-1\right) & \text { for } t=\hat{\tau}_{n+1}, N_{t}^{-}-N_{t-}^{-}=1 .\end{cases}
\end{aligned}
$$

Hence condition (3) is satisfied if and only if (4) holds.

Next, we examine efficient hedging strategies for a given European claim with maturity $S$ and payoff $F\left(X_{S}\right) \geq-c$, assuming $F(\cdot)$ is a continuous function.

Let $\mathcal{M}$ denote the family of all equivalent martingale measures for $X$. The superhedge price for the option $F$ at time $t<S$ admits the representation

$$
F_{u, d}:=\sup _{Q \in \mathcal{M}} E_{Q}\left[F\left(X_{S}\right) \mid N_{t}^{+}=u, N_{t}^{-}=d\right] ;
$$

see [7] and [17]. It does not depend on $t$ since, given $t$, we can find a measure $P^{\prime}$ equivalent to $P$ such that $N^{i}$ has constant intensity $\lambda^{i} \frac{S}{S-t}, i=+,-$. The name superhedge price is derived from the fact that, if the capital/wealth available to an investor at time $t$ is $V_{t} \geq F_{u, d}$, then there exists a self-financing strategy (see (2)) such that the capital/wealth at maturity satisfies $V_{S} \geq F\left(X_{S}\right)$ a.s. Clearly, the model specified by (1) is incomplete. Especially, it can be shown that the superhedge price for a European call option with payoff $F\left(X_{S}\right)=\left(X_{S}-K\right)^{+}$is given by $x_{0}$ for any maturity $S$ and strike $K$ (see, e.g., [11]). This price allows for arbitrage for the seller of the option. This example illustrates that superhedging may not be appropriate in model (1).

A feasible efficient strategy depends on the investors attitude towards risk. For our purposes, this attitude is incorporated in the choice of a loss function $l$ such that $l$ is increasing, convex, and $l(z)=0$ for $z \leq 0$. A typical choice is $l(z)=z^{p}$ for $z \geq 0$. Here the parameter $p \geq 1$ corresponds directly to the investor's degree of risk aversion. We shall assume that

$$
E\left[l\left(F\left(X_{S}\right)+c\right)\right]<\infty
$$

holds.

As introduced in [8], an efficient hedging strategy $\xi^{*}$ is a solution to the optimization problem

$$
J_{0}^{*}:=\min _{\xi \in \mathcal{A}_{V_{0}}} E\left[l\left(F\left(X_{S}\right)-V_{0}-\int_{0}^{S} \xi_{s} d X_{s}\right)\right] .
$$

The main reason for this criterion is that, since the market is incomplete, no perfect replication is possible. On the other hand, superreplication is neither appropriate nor economical, and so one tolerates some risk that one wants to minimize while taking into account the investor's attitude towards risk. The minimal risk $J^{*}$ is called the value function (or optimal cost-to-go function). More generally, the value function at time $t$ is given by

(8) $J^{*}(v, u, d, t)=\min _{\xi \in \mathcal{A}_{v, u, d, t}} E\left[l\left(F\left(X_{S}\right)-v-\int_{t}^{S} \xi_{s} d X_{s}\right) \mid N_{t}^{+}=u, N_{t}^{-}=d\right]$, 
where $\mathcal{A}_{v, u, d, t}$ denotes the class of admissible strategies on the interval $[t, S]$ given $V_{t}=v, N_{t}^{+}=u$, and $N_{t}^{-}=d$. The value $J^{*}(v, u, d, t)$ represents the optimal minimal value at time $t$ over the remaining period when the current information corresponds to $V_{t}=v, N_{t}^{+}=u$, and $N_{t}^{-}=d$. In line with the comment after (5) notice also that, if at any time $t$ one has $v \geq F_{u, d}$ with $F_{u, d}$ as in (5), then $J^{*} \equiv 0$. The optimization problem as such is therefore meaningful only if $v<F_{u, d}$. In what follows we shall consider for $v$ the entire closed interval $\left[-c, F_{u, d}\right]$ since we want to obtain the hedging strategy also for $v=F_{u, d}$. In this situation, and up to the first jump after $t$, the interval in (4) is given by

$$
I_{v, u, d}:=\left[-\frac{c+v}{x_{0} e^{a u-b d}\left(e^{a}-1\right)}, \frac{c+v}{x_{0} e^{a u-b d}\left(1-e^{-b}\right)}\right] .
$$

We gather two facts about the structure of the model in the next lemma.

LEMMA 2.2 .

(i) The number $N_{t}:=N_{t}^{+}+N_{t}^{-}$of jumps up to time $t$ is a Poisson process with intensity $\lambda=\lambda^{+}+\lambda^{-}$

(ii) For any nonnegative function $f$ we have

$$
\begin{aligned}
E[ & \left.f\left(N_{\hat{\tau}_{u+d+1}}^{+}, N_{\hat{\tau}_{u+d+1}}^{-}, \hat{\tau}_{u+d+1}\right) \mid N_{t}^{+}=u, N_{t}^{-}=d\right] \\
= & \int_{0}^{S-t}\left\{\lambda^{+} f(u+1, d, t+s)+\lambda^{-} f(u, d+1, t+s)\right\} e^{-\lambda s} d s \\
& +e^{-\lambda(S-t)} f(u, d, S) .
\end{aligned}
$$

Remark 1. The expression in (10) represents the expected value of $f$, taking into account that there may be a next jump either upwards or downwards or no further jump at all and given that at the current time $t$ one has observed $u$ jumps upwards and $d$ downwards.

Proof. Only item (ii) requires a proof. To simplify notation, we assume, without loss of generality, $u=d=t=0$. Let $\tau_{1}^{+}$(respectively, $\tau_{1}^{-}$) denote the time of the first jump up (respectively, down). We then have

$$
\begin{aligned}
E\left[f\left(N_{\hat{\tau}_{1}}^{+}, N_{\hat{\tau}_{1}}^{-}, \hat{\tau}_{1}\right), \tau_{1} \leq S\right]= & E\left[f\left(1,0, \tau_{1}^{+}\right), \tau_{1}^{+}<\tau_{1}^{-}, \tau_{1}^{+} \leq S\right] \\
& +E\left[f\left(0,1, \tau_{1}^{-}\right), \tau_{1}^{-}<\tau_{1}^{+}, \tau_{1}^{-} \leq S\right] \\
= & E\left[f\left(1,0, \tau_{1}^{+}\right) P\left[\tau_{1}^{+}<\tau_{1}^{-}, \tau_{1}^{+} \leq S \mid \tau_{1}^{+}\right]\right] \\
& +E\left[f\left(0,1, \tau_{1}^{-}\right) P\left[\tau_{1}^{-}<\tau_{1}^{+}, \tau_{1}^{-} \leq S \mid \tau_{1}^{-}\right]\right] \\
= & \int_{0}^{S} f(1,0, t) P\left[t<\tau_{1}^{-}\right] P\left[\tau_{1}^{+} \in d t\right] \\
& +\int_{0}^{S} f(0,1, t) P\left[t<\tau_{1}^{+}\right] P\left[\tau_{1}^{-} \in d t\right] \\
= & \int_{0}^{S} f(1,0, t) e^{-\lambda^{-} t} \lambda^{+} e^{-\lambda^{+} t} d t \\
& +\int_{0}^{S} f(0,1, t) e^{-\lambda^{+} t} \lambda^{-} e^{-\lambda^{-} t} d t .
\end{aligned}
$$

Adding to this expression the term

$$
E\left[f\left(N_{\hat{\tau}_{1}}^{+}, N_{\hat{\tau}_{1}}^{-}, \hat{\tau}_{1}\right), \tau_{1}>S\right]=e^{-\lambda S} f(0,0, S),
$$

we arrive at (10). 
3. Known intensities. We first examine the structure of the efficient strategy in the case where the investor has no doubt regarding the true values of the jump intensities $\lambda^{+}, \lambda^{-}$.

3.1. The PD-DP equation. In our situation, the state space is given by

$$
E=\{(v, u, d, t) \mid v \geq-c, u, d \in \mathbb{N}, t \in[0, S]\} .
$$

Let $\mathcal{C}(E)$ denote the class of all functionals $J$ on $E$ such that $(v, t) \mapsto J(v, u, d, t)$ is continuous for all $u, d \in \mathbb{N}$. We endow $\mathcal{C}(E)$ with the supremum norm

$$
\|J\|=\sup _{(v, u, d, t) \in E}|J(v, u, d, t)| .
$$

The process $X$ is piecewise deterministic. Hence problem (8) is a piecewise deterministic control problem. We only remark that, although the price process $X$ is piecewise constant, the optimal control will, in general, not be piecewise constant. This is due to the fact that if $X$ remains constant from time $t$ to time $t+s$, the time horizon changes, and thus the optimal strategy needs to be adapted.

For $J: E \rightarrow \mathbb{R}^{+}$, we define the operator $T$ mapping $J$ to $T J: E \rightarrow \mathbb{R}^{+}$by

$$
\begin{aligned}
& (T J)(v, u, d, t) \\
& =\int_{0}^{S-t} e^{-\lambda s} \cdot \min _{\zeta \in I_{v, u, d}}\left\{\begin{array}{c}
\lambda^{+} J\left(v+\zeta x_{0} e^{a u-b d}\left(e^{a}-1\right), u+1, d, t+s\right) \\
+\lambda^{-} J\left(v+\zeta x_{0} e^{a u-b d}\left(e^{-b}-1\right), u, d+1, t+s\right)
\end{array}\right\} d s \\
& \quad+e^{-\lambda(S-t)} l\left(F\left(x_{0} e^{a u-b d}\right)-v\right),
\end{aligned}
$$

where $I_{v, u, d}$ is as in (9). In (11) one takes the min over the present control actions of the expectation of the optimal cost-to-go function at the next jump time, where one of the two possibilities may occur: a jump upwards for which the value process changes to $v+\zeta x_{0} e^{a u-b d}\left(e^{a}-1\right)$ or a jump downwards (see also the proof of Lemma 3.2 and the explicit expressions (20) and (21) of $J^{1}$ and $J^{2}$ after its proof). From the proof of the next lemma it follows that the integral in (11) is well defined: indeed, the integrand is given by the continuous function $\hat{g}$ defined in (12).

LEMmA 3.1. The operator $T: \mathcal{C}(E) \rightarrow \mathcal{C}(E)$ is a contraction with contraction constant $1-e^{-\lambda S}$.

Proof. 1. We first demonstrate that, for $J \in \mathcal{C}(E)$, the integral in (11) is well defined and $T J \in \mathcal{C}(E)$. Let

$$
\begin{aligned}
g(\zeta, v, s)= & \lambda^{+} J\left(v+\zeta x_{0} e^{a u-b d}\left(e^{a}-1\right), u+1, d, t+s\right) \\
& +\lambda^{-} J\left(v+\zeta x_{0} e^{a u-b d}\left(e^{-b}-1\right), u, d+1, t+s\right)
\end{aligned}
$$

denote the function inside the curly brackets in (11). This function is continuous on

$$
\left[-\frac{c+F_{u, d}}{x_{0} e^{a u-b d}\left(e^{a}-1\right)}, \frac{c+F_{u, d}}{x_{0} e^{a u-b d}\left(1-e^{-b}\right)}\right] \times\left[-c, F_{u, d}\right] \times[0, T]
$$

hence it is also bounded on this domain. The multifunction $v \mapsto I_{v, u, d}$ is continuous. Applying Proposition D.3 (c) of [12], we obtain that the function

$$
\hat{g}(v, s)=\min _{z \in I_{v, u, d}} g(z, v, s)
$$

is continuous. 
2. The proof that $T$ is a contraction is inspired by Theorem 3.2 of [1]. For $\xi$ : $[0, S-t] \rightarrow I_{v, u, d}$, let

$$
\begin{aligned}
\left(T_{\xi} J\right)(v, u, d, t)= & e^{-\lambda(S-t)} l\left(F\left(x_{0} e^{a u-b d}\right)-v\right) \\
+\int_{0}^{S-t} e^{-\lambda s}\{ & \lambda^{+} J\left(v+\xi(s) x_{0} e^{a u-b d}\left(e^{a}-1\right), u+1, d, t+s\right) \\
& \left.\quad+\lambda^{-} J\left(v+\xi(s) x_{0} e^{a u-b d}\left(e^{-b}-1\right), u, d+1, t+s\right)\right\} d s .
\end{aligned}
$$

We then have

$$
\left\|T_{\xi} J-T_{\xi} J^{\prime}\right\| \leq\left(1-e^{-\lambda S}\right)\left\|J-J^{\prime}\right\| .
$$

Due to the continuity of $J$, there exists $\xi:[0, S-t] \rightarrow I_{v, u, d}$ such that

$$
T_{\xi} J=T J
$$

Hence we obtain

$$
\begin{aligned}
T J^{\prime}-T J & \leq T_{\xi} J^{\prime}-T_{\xi} J \\
& \leq\left(1-e^{-\lambda S}\right)\left\|J^{\prime}-J\right\| .
\end{aligned}
$$

By symmetry we can conclude that

$$
\left|T J^{\prime}-T J\right| \leq\left(1-e^{-\lambda S}\right)|| J^{\prime}-J \| .
$$

Given $n \in \mathbb{N}$, let

$$
J^{0}=0, \quad \text { and, for } h \leq n, \quad J^{h}=T J^{h-1},
$$

and let $\left(\xi_{s}^{n}\right)_{s \in[0, S]}$ be the strategy induced by computing $J^{n}\left(V_{0}, 0,0,0\right)$ via (13) and (11). More precisely, this strategy is defined as follows. By induction over $h \leq n-2$ we define $\xi^{n}$ on each interval $\left(\hat{\tau}_{h}, \hat{\tau}_{h+1}\right]$ : For $s \in\left[0, \hat{\tau}_{1}\right]$ let $v=V_{0}$ and

$\xi_{s}^{n}:=\arg \min _{\zeta \in I_{v, 0,0}}\left\{\lambda^{+} J^{n-1}\left(v+\zeta x_{0}\left(e^{a}-1\right), 1,0, s\right)+\lambda^{-} J^{n-1}\left(v+\zeta x_{0}\left(e^{-b}-1\right), 0,1, s\right)\right\}$.

It was shown in [8] that the mapping $v \mapsto J^{n}(v, u, d, t)$ is decreasing and strictly convex on $\left(-c, F_{u, d}\right)$. Hence the minimum in (14) and (15) below is assumed at a unique point $\zeta$.

Suppose we defined the strategy $\xi_{s}^{n}$ for all $s \leq \hat{\tau}_{h}$. At time $t=\hat{\tau}_{h}$, we observed $u=N_{t}^{+}$jumps up and $d=N_{t}^{-}$jumps down with $u+d=h$, and we have some capital $v=V_{\hat{\tau}_{h}}$ available. For $s \in\left(\hat{\tau}_{h}, \hat{\tau}_{h+1}\right]$, we define

$$
\begin{aligned}
\xi_{s}^{n}:=\arg \min _{\zeta \in I_{v, u, d}}\{ & \lambda^{+} J^{n-u-d-1}\left(v+\zeta x_{0} e^{a u-b d}\left(e^{a}-1\right), u+1, d, s\right) \\
& \left.+\lambda^{-} J^{n-u-d-1}\left(v+\zeta x_{0} e^{a u-b d}\left(e^{-b}-1\right), u, d+1, s\right)\right\} .
\end{aligned}
$$

We have thus defined the strategy $\xi_{t}^{n}$ for $t \in\left[0, \hat{\tau}_{n-1}\right]$. On the event $\left\{\tau_{n-1}>S\right\}$, this is sufficient. For the general case, we set $\xi_{t}^{n}=0$ for $t \in\left(\hat{\tau}_{n-1}, S\right]$; i.e., we transfer all funds to the cash account after the $(n-1)$ st jump happened prior to $S$. 
Clearly, the strategy $\xi^{n}$ thus defined satisfies $\xi^{n} \in \mathcal{A}_{V_{0}}$. The associated wealth process

$$
V_{t}^{n}:=V_{0}+\int_{0}^{t} \xi_{s}^{n} d X_{s}
$$

is constant from time $\hat{\tau}_{n-1}$ on.

By analogy to (8), we define a new value function

$$
J^{*, n}(v, u, d, t)=\min _{\xi \in \mathcal{A}_{v, u, d, t}} E\left[l\left(F\left(X_{S}\right)-V_{S}^{\xi}\right), \tau_{u+d+n}>S \mid N_{t}^{+}=u, N_{t}^{-}=d\right],
$$

where

$$
V_{S}^{\xi}=v+\int_{t}^{S} \xi_{s} d X_{s}
$$

The difference is that for $J^{*, n}(v, u, d, t)$ we take the expectation on the event that less than $n$ jumps occur on the interval $(t, S]$.

We have now defined three functions $J^{*}, J^{n}$, and $J^{*, n}$ via (8), (13), and (17). Of these, $J^{*}$ is the value function associated with problem (7). The function $J^{n}$ is defined as the $n$th iteration of the dynamic programming operator associated with problem (7). Hence, a priori, $J^{n}$ is not a value function. However, $J^{*, n}$ is the value function for the problem obtained from (7) by replacing $\Omega$ by $\Omega \cap\left\{\tau_{n}>S\right\}$. In the next lemma, we demonstrate that $J^{n}$ and $J^{*, n}$ coincide. Consequently, we obtain for $t=0$ the equality

$J_{0}^{n}:=J^{n}\left(V_{0}, 0,0,0\right)=E\left[l\left(F\left(X_{S}\right)-V_{S}^{n}\right), \tau_{n}>S\right]=\min _{\xi \in \mathcal{A}_{V_{0}}} E\left[l\left(F\left(X_{S}\right)-V_{S}^{\xi}\right), \tau_{n}>S\right]$

with $V^{n}$ defined as in (16). This is remarkable in that it gives a new interpretation for $J^{n}$ as a value function to problem (17) which is related to the original problem in a simple intuitive way.

LEMma 3.2. We have $J^{n}=J^{*, n}$.

Proof. Essentially, the assertion is an application of the DP principle, Proposition 2.1 , and (10).

For $n=0,1$, the assertion is immediate, see also (20).

Assume $J^{*, n-1}=J^{n-1}$ holds for $n-1 \in \mathbb{N}$. We now demonstrate the assertion for $n$. Conditioning the right-hand side of (17) on $\hat{\tau}_{u+d+1}$, we obtain from the DP principle that

$$
\begin{aligned}
J^{*, n} & (v, u, d, t) \\
& =\min _{\xi \in \mathcal{A}_{v, u, d, t}} E\left[J^{*, n-1}\left(V_{\hat{\tau}_{u+d+1}}, N_{\hat{\tau}_{u+d+1}}^{+}, N_{\hat{\tau}_{u+d+1}}^{-}, \hat{\tau}_{u+d+1}\right) \mid N_{t}^{+}=u, N_{t}^{-}=d\right] \\
& =\min _{\xi \in \mathcal{A}_{v, u, d, t}} E\left[J^{n-1}\left(V_{\hat{\tau}_{u+d+1}}, N_{\hat{\tau}_{u+d+1}}^{+}, N_{\hat{\tau}_{u+d+1}}^{-}, \hat{\tau}_{u+d+1}\right) \mid N_{t}^{+}=u, N_{t}^{-}=d\right] .
\end{aligned}
$$

Due to (10) and Proposition 2.1, the right-hand side of the previous equation evaluates to

$$
\begin{aligned}
& \min _{\xi \in \mathcal{A}_{v, u, d, t}} \int_{0}^{S-t} e^{-\lambda s}\left\{\begin{array}{r}
\lambda^{+} J^{n-1}\left(v+\xi_{s} x_{0} e^{a u-b d}\left(e^{a}-1\right), u+1, d, t+s\right) \\
+\lambda^{-} J^{n-1}\left(v+\xi_{s} x_{0} e^{a u-b d}\left(e^{-b}-1\right), u, d+1, t+s\right)
\end{array}\right\} d s \\
& +e^{-\lambda(S-t)} l\left(F\left(x_{0} e^{a u-b d}\right)-v\right)
\end{aligned}
$$




$$
\begin{aligned}
&= \int_{0}^{S-t} e^{-\lambda s} \min _{\zeta \in I_{v, u, d}}\left\{\begin{array}{r}
\lambda^{+} J^{n-1}\left(v+\zeta x_{0} e^{a u-b d}\left(e^{a}-1\right), u+1, d, t+s\right) \\
+\lambda^{-} J^{n-1}\left(v+\zeta x_{0} e^{a u-b d}\left(e^{-b}-1\right), u, d+1, t+s\right)
\end{array}\right\} d s \\
&+e^{-\lambda(S-t)} l\left(F\left(x_{0} e^{a u-b d}\right)-v\right),
\end{aligned}
$$

which proves the assertion for $n$.

We have explicit expressions for the first two iterations of $T$ :

$$
\begin{aligned}
J^{1}(v, u, d, t)= & e^{-\lambda(S-t)} l\left(F\left(x_{0} e^{a u-b d}\right)-v\right) \\
J^{2}(v, u, d, t)= & J^{1}(v, u, d, t) \\
& +(S-t) \min _{\zeta \in I_{v, u, d}}\left\{\begin{array}{c}
\lambda^{+} J^{1}\left(v+\zeta x_{0} e^{a u-b d}\left(e^{a}-1\right), u+1, d, t\right) \\
+\lambda^{-} J^{1}\left(v+\zeta x_{0} e^{a u-b d}\left(e^{-b}-1\right), u, d+1, t\right)
\end{array}\right\},
\end{aligned}
$$

where we have used the fact that $J^{1}$ depends on $t$ only through the factor given by the exponential function. Obtaining an explicit expression of $J^{n}(v, u, d, t)$ becomes difficult, if not impossible, for $n>2$, unless one makes some simplifying assumptions that would, however, lead to a suboptimal solution. In section 5 we shall therefore describe a computable approximation approach and show its convergence.

THEOREM 3.3.

(i) The value function $J^{*}$ is the unique fixed point of $T$, i.e.,

$$
J^{*}=T J^{*},
$$

and we have

$$
\left\|J^{n}-J^{*}\right\| \leq e^{\lambda S}\left(1-e^{-\lambda S}\right)^{n}\left\|J^{1}\right\| .
$$

(ii) The following strategy $\xi^{*}$ is efficient: For $s \in\left(\hat{\tau}_{u+d}, \hat{\tau}_{u+d+1}\right]$ and $v=V_{\tau_{u+d}}$, let $\xi_{s}^{*}$ be given by the unique solution to the deterministic optimization problem embedded in the computation of $\left(T J^{*}\right)(u, d, v, t)$ according to (11), i.e.,

$$
\begin{aligned}
\xi_{s}^{*}:=\arg \min _{\zeta \in I_{v, u, d}}\{ & \lambda^{+} J^{*}\left(v+\zeta x_{0} e^{a u-b d}\left(e^{a}-1\right), u+1, d, s\right) \\
& \left.+\lambda^{-} J^{*}\left(v+\zeta x_{0} e^{a u-b d}\left(e^{-b}-1\right), u, d+1, s\right)\right\} .
\end{aligned}
$$

Proof. From (18) we obtain

$$
J_{0}^{n} \leq J_{0}^{*} \leq J_{0}^{n}+E\left[l\left(F\left(X_{S}\right)+c\right), \tau_{n} \leq S\right] .
$$

Due to estimates (24) and (6), we have

$$
\lim _{n \uparrow \infty} J^{n}(v, 0,0, t)=J^{*}(v, 0,0, t)
$$

uniformly in $v$ and $t$. Since $N^{+}, N^{-}$are stationary Markov processes, we obtain $J^{n}(v, u, d, t) \rightarrow J^{*}(v, u, d, t)$ uniformly in $v$ and $t$ for all $u, d$. This implies $J^{*} \in \mathcal{C}(E)$; i.e., $J^{*}$ is in the domain of $T$. Application of the DP principle implies that $J^{*}$ is a fixed point of $T$ (alternatively, this follows from (25) and Lemma 3.1).

For item (ii), we obtain from the DP principle

$$
\begin{gathered}
J^{*}(v, u, d, t)=\min _{\xi \in \mathcal{A}_{v, u, d, t}} E\left[J^{*}\left(v+\int_{t}^{\tau_{u+d+1}} \xi_{s} d X_{s}, N_{\tau_{u+d+1}}^{+}, N_{\tau_{u+d+1}}^{-}, \tau_{u+d+1}\right) \mid\right. \\
\left.N_{t}^{+}=u, N_{t}^{-}=d\right] .
\end{gathered}
$$


Due to (10), the right-hand side of the previous equation evaluates to

$$
\begin{aligned}
& \min _{\xi \in \mathcal{A}_{v, u, d, t}} \int_{0}^{S-t} e^{-\lambda s}\left\{\begin{array}{c}
\lambda^{+} J^{*}\left(v+\xi_{s} x_{0} e^{a u-b d}\left(e^{a}-1\right), u+1, d, t+s\right) \\
+\lambda^{-} J^{*}\left(v+\xi_{s} x_{0} e^{a u-b d}\left(e^{-b}-1\right), u, d+1, t+s\right)
\end{array}\right\} d s \\
& +e^{-\lambda(S-t)} l\left(F\left(x_{0} e^{a u-b d}\right)-v\right) .
\end{aligned}
$$

The minimum is achieved by the strategy $\xi^{*}$ prescribed in item (ii) (see also (19)). This proves optimality of the strategy $\xi^{*}$, and we obtain again the fixed-point equation

$$
J^{*}(v, u, d, t)=\left(T J^{*}\right)(v, u, d, t) .
$$

Remark 2. In computing the approximating strategy $\xi^{n},(23)$ gives a handle on the distance between the value of the $n$th iteration $J_{0}^{n}$ and the optimal value $J_{0}^{*}$. However, from an economic point of view, one is equally interested in the expected loss incurred from implementing the strategy $\xi^{n}$ associated with this iteration, i.e., in the term $E\left[l\left(F\left(X_{S}\right)-V_{S}^{n}\right)\right]$, where, we recall from (16), $V_{t}^{n}$ is the wealth process associated with the strategy $\xi^{n}$. This can be estimated via

$$
J_{0}^{n} \leq E\left[l\left(F\left(X_{S}\right)-V_{S}^{n}\right)\right] \leq J_{0}^{n}+E\left[l\left(F\left(X_{S}\right)+c\right), \tau_{n} \leq S\right]
$$

which follows from (18). In section 5 , we examine the numerical implementation of the problem in more detail.

\section{Uncertain intensities.}

4.1. Bayesian updating. In this section, we assume $\lambda^{+}$and $\lambda^{-}$are constant but unknown to the investor; i.e., the true probability distribution $P=P_{\lambda^{+}, \lambda^{-}}$is unknown to the investor. In modeling this situation we take the Bayesian point of view, thereby assuming that $\lambda^{+}$and $\lambda^{-}$are random variables to which the investor assigns some (prior) distributions $\pi_{0}^{+}\left(d \lambda^{+}\right)$and $\pi_{0}^{-}\left(d \lambda^{-}\right)$. Let $(\Omega, \mathcal{F}, P)$ denote the given probability space where $P=P_{\lambda^{+}, \lambda^{-}}$. On $\bar{\Omega}:=\mathbb{R}_{+}^{2} \times \Omega$, the subjective probability measure of the investor is thus given by

$$
P(d \bar{\omega})=\pi_{0}^{+}\left(d \lambda^{+}\right) \pi_{0}^{-}\left(d \lambda^{-}\right) P_{\lambda^{+}, \lambda^{-}}(d \omega) .
$$

We consider two filtrations on $\bar{\Omega}$ :

(i) The filtration $\left(\mathcal{F}_{t}\right)$ generated by the processes $N^{+}$and $N^{-}$. This is the information available to the investor.

(ii) Let $\mathcal{G}_{0}$ denote the $\sigma$-algebra generated by $\lambda^{+}$and $\lambda^{-}$. We then define the filtration $\mathcal{G}_{t}=\mathcal{F}_{t} \vee \mathcal{G}_{0}$ as the filtration corresponding to "full information."

We have that, with respect to the filtration $\left(\mathcal{F}_{t}\right), N$ is a Cox process, whereas, with respect to the filtration $\left(\mathcal{G}_{t}\right), N$ is a Poisson process with intensity $\lambda^{+}+\lambda^{-}$.

We define $P_{0}$ via

$$
\left.\frac{d P}{d P_{0}}\right|_{\mathcal{G}_{t}}=e^{-\left(\lambda^{+}+\lambda^{-}-2\right) t}\left(\lambda^{+}\right)^{N_{t}^{+}}\left(\lambda^{-}\right)^{N_{t}^{-}}=: L_{t} .
$$

Under $P_{0}$, the random quantities $\lambda^{+}, \lambda^{-}, N^{+}$, and $N^{-}$are independent: $N^{+}$and $N^{-}$are standard Poisson processes; i.e., both have known intensity one. The random variables $\lambda^{i}$ have distribution $\pi_{0}^{i}$ under $P_{0}$ for $i=+,-$. Let

$$
\begin{aligned}
\mathcal{L}_{t}(f) & :=E_{0}\left[L_{t} f\left(\lambda^{+}, \lambda^{-}\right) \mid \mathcal{F}_{t}\right] \\
& =\int_{0}^{\infty} \int_{0}^{\infty} f\left(\lambda^{+}, \lambda^{-}\right) e^{-\left(\lambda^{+}+\lambda^{-}-2\right) t}\left(\lambda^{+}\right)^{N_{t}^{+}}\left(\lambda^{-}\right)^{N_{t}^{-}} \pi_{0}^{+}\left(d \lambda^{+}\right) \pi_{0}^{-}\left(d \lambda^{-}\right)
\end{aligned}
$$


and put

$$
\pi_{t}^{i}(d \lambda):=\frac{e^{-\lambda t} \lambda^{N_{t}^{i}} \pi^{i}(d \lambda)}{\int_{0}^{\infty} e^{-\lambda t} \lambda^{N_{t}^{i}} \pi^{i}(d \lambda)}, \quad i=+,-.
$$

We then have the relation (filter equation)

$$
E\left[f\left(\lambda^{+}, \lambda^{-}\right) \mid \mathcal{F}_{t}\right]=\frac{\mathcal{L}_{t}(f)}{\mathcal{L}_{t}(1)}=\iint f\left(\lambda^{+}, \lambda^{-}\right) \pi_{t}^{+}\left(d \lambda^{+}\right) \pi_{t}^{-}\left(d \lambda^{-}\right),
$$

and $\pi_{t}^{i}\left(d \lambda^{i}\right)$ is the posterior distribution of $\lambda^{i}, i=+,-$.

Recall that the density of the gamma distribution with shape parameter $\alpha$ and scale parameter $\beta$ can be given in the form

$$
\gamma(\lambda \mid \alpha, \beta)=\frac{\beta^{\alpha}}{\Gamma(\alpha)} \lambda^{\alpha-1} e^{-\beta \lambda} .
$$

We conclude from (27) that, if the prior distribution $\pi_{0}^{i}$ for $\lambda^{i}$ is a gamma distribution with parameters $\alpha_{0}^{i}$ and $\beta_{0}$, then the posterior distribution $\pi_{t}$ at time $t$ is again a gamma distribution with parameters

$$
\alpha_{t}^{i}=\alpha_{0}^{i}+N_{t}^{i}, \quad \beta_{t}=\beta_{0}+t
$$

for $i=+,-$. Furthermore, the distribution of $\lambda=\lambda^{+}+\lambda^{-}$at time 0 is gamma with parameters $\alpha_{0}=\alpha_{0}^{+}+\alpha_{0}^{-}$and $\beta_{0}$. At time $t$, the posterior distribution of $\lambda$ is again gamma with $\beta_{t}$ as in (29) and

$$
\alpha_{t}=\alpha_{t}^{+}+\alpha_{t}^{-}=\alpha_{0}+N_{t} .
$$

We can calculate by means of $(28)$

$$
\overline{\lambda_{t}^{i}}:=E\left[\lambda^{i} \mid \mathcal{F}_{t}\right]=\frac{\alpha_{t}^{i}}{\beta_{t}}, \quad i=+,-.
$$

4.2. The PD-DP equation. The observed process $X$ is still piecewise deterministic. Hence problem (8) is still a piecewise deterministic control problem. In the case of uncertain intensities, if $X$ remains constant from time $t$ to time $t+s$, this not only changes the time to maturity, but it also reveals additional information regarding the true intensities.

Let

$$
\begin{aligned}
p^{+}(u, d, t, s) & :=\left(\frac{\beta_{0}+t}{\beta_{0}+t+s}\right)^{\alpha_{0}+u+d} \frac{\alpha_{0}^{+}+u}{\beta_{0}+t+s}, \\
p^{-}(u, d, t, s) & :=\left(\frac{\beta_{0}+t}{\beta_{0}+t+s}\right)^{\alpha_{0}+u+d} \frac{\alpha_{0}^{-}+d}{\beta_{0}+t+s}, \\
p^{0}(n, t) & :=\left(\frac{\beta_{0}+t}{\beta_{0}+S}\right)^{\alpha_{0}+n} .
\end{aligned}
$$

Lemma 4.1. For any nonnegative function $f$, we have

$$
\begin{aligned}
E[ & \left.f\left(N_{\hat{\tau}_{u+d+1}}^{+}, N_{\hat{\tau}_{u+d+1}}^{-}, \hat{\tau}_{u+d+1}\right) \mid N_{t}^{+}=u, N_{t}^{-}=d\right] \\
= & \int_{0}^{S-t}\left\{p^{+}(u, d, t, s) f(u+1, d, t+s)+p^{-}(u, d, t, s) f(u, d+1, t+s)\right\} d s \\
& +p^{0}(u+d, t) f(u, d, S) .
\end{aligned}
$$


Proof. Since $N$ is a Poisson process with intensity $\lambda^{+}+\lambda^{-}$with respect to the filtration $\left(\mathcal{G}_{t}\right)$, we obtain from $(10)$

$$
\begin{aligned}
& E\left[f\left(N_{\hat{\tau}_{u+d+1}}^{+}, N_{\hat{\tau}_{u+d+1}}^{-}, \hat{\tau}_{u+d+1}\right) \mid N_{t}^{+}=u, N_{t}^{-}=d\right] \\
& =E\left[E\left[f\left(N_{\hat{\tau}_{u+d+1}}^{+}, N_{\hat{\tau}_{u+d+1}}^{-}, \hat{\tau}_{u+d+1}\right) \mid \mathcal{G}_{t}\right] \mid N_{t}^{+}=u, N_{t}^{-}=d\right] \\
& =E\left[\int_{0}^{S-t}\left\{\lambda^{+} f(u+1, d, t+s)+\lambda^{-} f(u, d+1, t+s)\right\} e^{-\lambda s} d s\right. \\
& \left.\quad+e^{-\lambda(S-t)} f(u, d, S) \mid N_{t}^{+}=u, N_{t}^{-}=d\right] .
\end{aligned}
$$

Due to (28) and (29), the last expression is given by

$$
\begin{aligned}
& \iint \gamma\left(\lambda^{+} \mid \alpha_{0}^{+}+u, \beta_{0}+t\right) \gamma\left(\lambda^{-} \mid \alpha_{0}^{-}+d, \beta_{0}+t\right) d \lambda^{+} d \lambda^{-} \\
& \cdot\left[\int_{0}^{S-t}\left\{\lambda^{+} f(u+1, d, t+s)+\lambda^{-} f(u, d+1, t+s)\right\} e^{-\lambda s} d s+e^{-\lambda(S-t)} f(u, d, S)\right] .
\end{aligned}
$$

Applying Fubini's theorem to integrate first over $\lambda^{+}, \lambda^{-}$and then over $s$, we arrive at the right-hand side of (32).

In the case of uncertain intensities, the DP operator $T$ maps $J: E \rightarrow \mathbb{R}^{+}$to $T J: E \rightarrow \mathbb{R}^{+}$defined by

$$
\begin{aligned}
& (T J)(v, u, d, t) \\
& =\int_{0}^{S-t} \min _{\zeta \in I_{v, u, d}}\left\{\begin{array}{l}
p^{+}(u, d, t, s) J\left(v+\zeta x_{0} e^{a u-b d}\left(e^{a}-1\right), u+1, d, t+s\right) \\
+p^{-}(u, d, t, s) J\left(v+\zeta x_{0} e^{a u-b d}\left(e^{-b}-1\right), u, d+1, t+s\right)
\end{array}\right\} d s \\
& \quad+p^{0}(u+d, t) l\left(F\left(x_{0} e^{a u-b d}\right)-v\right) .
\end{aligned}
$$

Let

$$
E^{k}=\{(v, u, d, t) \mid v \geq-c, u, d \in \mathbb{N}, u+d \leq k, t \in[0, S]\}
$$

and

$$
\left(T^{k} J\right)(v, u, d, t):= \begin{cases}(T J)(v, u, d, t), & u+d \leq k-1, \\ 0, & u+d=k .\end{cases}
$$

Lemma 4.2. For every $k \in \mathbb{N}$, the operator $T^{k}: \mathcal{C}\left(E^{k}\right) \rightarrow \mathcal{C}\left(E^{k}\right)$ is a contraction with contraction constant

$$
1-p^{0}(k, 0)=1-\left(\frac{\beta_{0}}{\beta_{0}+S}\right)^{\alpha_{0}+k}
$$

and $T^{k}$ has a unique fixed point in $\mathcal{C}\left(E^{k}\right)$.

Proof. 1. It follows as in Lemma 3.1 that $T^{k} J \in \mathcal{C}\left(E^{k}\right)$ for $J \in \mathcal{C}\left(E^{k}\right)$.

2. For $\xi:[0, S-t] \rightarrow I_{v, u, d}$ with values denoted, as previously, by $\zeta$, let

$$
\begin{aligned}
\left(T_{\xi} J\right)(v, u, d, t)= & p^{0}(u+d, t) l\left(F\left(x_{0} e^{a u-b d}\right)-v\right) \\
& +\int_{0}^{S-t}\left\{p^{+}(u, d, t, s) J\left(v+\zeta(s) x_{0} e^{a u-b d}\left(e^{a}-1\right), u+1, d, t+s\right)\right. \\
& \left.+p^{-}(u, d, t, s) J\left(v+\zeta(s) x_{0} e^{a u-b d}\left(e^{-b}-1\right), u, d+1, t+s\right)\right\} d s .
\end{aligned}
$$


We then have

$$
\begin{aligned}
\left\|T_{\xi} J-T_{\xi} J^{\prime}\right\|_{E^{k}} & \leq\left\|\int_{0}^{S-t}\left\{p^{+}(u, d, t, s)+p^{-}(u, d, t, s)\right\} d s\right\|_{E^{k}}\left\|J-J^{\prime}\right\|_{E^{k}} \\
& =\left\|1-p^{0}(u+d, t)\right\|_{E^{k}}\left\|J-J^{\prime}\right\|_{E^{k}} \\
& =\left\|1-\left(\frac{\beta_{0}+t}{\beta_{0}+S}\right)^{\alpha_{0}+u+d}\right\|\left\|_{E^{k}}\right\| J-J^{\prime} \|_{E^{k}} \\
& \leq\left(1-\left(\frac{\beta_{0}}{\beta_{0}+S}\right)^{\alpha_{0}+k}\right)\left\|J-J^{\prime}\right\|_{E^{k}} .
\end{aligned}
$$

Due to the continuity of $J$, there exists $\xi:[0, S-t] \rightarrow I_{v, u, d}$ such that, for $u+d \leq k-1$,

$$
T_{\xi} J=T^{k} J
$$

Hence we obtain

$$
T^{k} J^{\prime}-T^{k} J \leq T_{\xi} J^{\prime}-T_{\xi} J \leq\left(1-\left(\frac{\beta_{0}}{\beta_{0}+S}\right)^{\alpha_{0}+k}\right)\left\|J^{\prime}-J\right\|_{E^{k}} .
$$

By symmetry we can conclude that

$$
\left\|T^{k} J^{\prime}-T^{k} J\right\|_{E^{k}} \leq\left(1-\left(\frac{\beta_{0}}{\beta_{0}+S}\right)^{\alpha_{0}+k}\right)\left\|J^{\prime}-J\right\|_{E^{k}} .
$$

Corollary 4.3. For $k \in \mathbb{N}$, a fixed point $J_{k}^{*}$ of $T^{k}$ coincides with a fixed point $J^{*}$ of $T$ on the set $E^{k}$.

Proof. Let $G=J^{*}$ on $E^{k-1}$ and $G=0$ on $E^{k} \backslash E^{k-1}$. It follows from (35) that $G$ is a fixed point of $T^{k}$. Due to Lemma 4.2, this implies $G=J_{k}^{*}$.

Remark 3. While the case of uncertain intensities follows in large part along the lines of known intensities, Lemma 4.2 highlights a major difference: The operator $T$ is no longer a contraction on $\mathcal{C}(E)$. This is due to the fact that, as the number of observed jumps increases, the estimated probability that no more jumps occur prior to $S$ tends to zero. But it is exactly this probability that makes $T$ a contraction; see also [1] for the case without model uncertainty.

For practical purposes, however, this poses no difficulties since (see Corollary 4.3) we can instead work with the contraction $T^{k}$, and for this see section 5 , especially Lemma 5.1.

We conclude this section by showing Theorem 4.5 below by which it follows that, despite the fact that in the present setting the operator $T$ is no longer a contraction, this operator has a unique fixed point. Furthermore, an efficient strategy exists in complete analogy to the case of known intensities.

As in (13), we define

$$
J^{0}=0, \quad \text { and, for } h \leq n, \quad J^{h}=T J^{h-1} .
$$

Again, we obtain explicit expressions for the first two iterations of $T$ (compare with (20) and (21)):

$$
\begin{aligned}
J^{1}(v, u, d, t)= & p^{0}(u+d, t) l\left(F\left(x_{0} e^{a u-b d}\right)-v\right), \\
J^{2}(v, u, d, t)= & J^{1}(v, u, d, t)+\frac{S-t}{\beta_{0}+S} \\
& \cdot \min _{\zeta \in I_{v, u, d}}\left\{\begin{array}{c}
\left(\alpha_{0}^{+}+u\right) J^{1}\left(v+\zeta x_{0} e^{a u-b d}\left(e^{a}-1\right), u+1, d, t\right) \\
+\left(\alpha_{0}^{-}+d\right) J^{1}\left(v+\zeta x_{0} e^{a u-b d}\left(e^{-b}-1\right), u, d+1, t\right)
\end{array}\right\} .
\end{aligned}
$$


In (38), we see directly how the observed jumps affect the optimal strategy: The larger the number $u$ of upward jumps in comparison to the number of downward jumps $d$, the higher the a posteriori probability that the next jump will be upwards. Concerning the explicit computation of the exact values of $J^{n}(v, u, d, t)$ for $n>2$ the same comments apply as after (21).

We define the strategy $\xi^{n}$ analogous to (14) and (15) in which $\lambda^{i}$ is replaced by $p^{i}(i=+,-)$.

LEMMA 4.4. We have

$$
J^{n}(v, u, d, t)=\min _{\xi \in \mathcal{A}_{v, u, d, t}} E\left[l\left(F\left(X_{S}\right)-V_{S}^{\xi}\right), \tau_{u+d+n}>S \mid N_{t}^{+}=u, N_{t}^{-}=d\right] .
$$

Proof. The proof proceeds exactly as in Lemma 3.2, with the exception that (10) is replaced by $(32)$.

THEOREM 4.5 .

(i) The value function $J^{*}$ is the unique fixed point of $T$, i.e.,

$$
J^{*}(v, u, d, t)=\left(T J^{*}\right)(v, u, d, t), \quad(v, u, d, t) \in E .
$$

(ii) The following strategy $\xi^{*}$ is efficient: For $s \in\left(\hat{\tau}_{u+d}, \hat{\tau}_{u+d+1}\right]$ and $v=V_{\tau_{u+d}}$, let $\xi_{s}^{*}=\zeta^{u, d, v, t, *}(s)$, where the latter is given by the deterministic optimization problem embedded in the computation of $\left(T J^{*}\right)(u, d, v, t)$, i.e.,

$$
\begin{aligned}
\zeta_{s}^{*}:=\arg \min _{\zeta \in I_{v, u, d}}\left\{p^{+}(u, d, t, s-t) J^{*}\left(v+\zeta x_{0} e^{a u-b d}\left(e^{a}-1\right), u+1, d, s\right)\right. \\
\left.+p^{-}(u, d, t, s-t) J^{*}\left(v+\zeta x_{0} e^{a u-b d}\left(e^{-b}-1\right), u, d+1, s\right)\right\} .
\end{aligned}
$$

Proof. We first prove (i), i.e., the uniqueness of the fixed point. Consider two fixed points $J^{a}$ and $J^{b}$ of $T$. Due to Corollary 4.3 and Lemma 4.2, we obtain

$$
J^{a}(v, u, d, t)=J^{b}(v, u, d, t), \quad(v, u, d, t) \in E^{k-1} .
$$

Since $k$ is arbitrary, this implies $J^{a}=J^{b}$ on $E$.

As in (24), we obtain

$$
J_{0}^{n} \leq J_{0}^{*} \leq J_{0}^{n}+E\left[l\left(F\left(X_{S}\right)+c\right), \tau_{n} \leq S\right] .
$$

From (40) and the uniqueness of the fixed point we obtain item (i) as in the proof of Theorem 3.3 .

Similarly, (ii) follows from the DP principle and (32). Especially, this implies that $J^{*}$ is a fixed point of $T$.

For a version of the estimate (23) in the case of uncertain intensities, we refer to Lemma 5.1.

We also have the following analogue to the estimate (26):

$$
J_{0}^{n} \leq E\left[l\left(F\left(X_{S}\right)-V_{S}^{n}\right)\right] \leq J_{0}^{n}+E\left[l\left(F\left(X_{S}\right)+c\right), \tau_{n} \leq S\right] .
$$

5. Algorithmic implementation: Interpolation of the value function. Due to Theorem 3.3 (respectively, 4.5), the value $J_{0}^{n}$ converges to the optimal value and the strategy $\xi^{n}$ to the efficient strategy in the sense of the estimate (26), respectively (41). In order to compute $J_{0}^{n}$ and $\xi^{n}$ for reasonably large values of $n$, we need to discretize the problem in the dimensions wealth and time $(v, t)$ and then to interpolate it in the same variables. In this section, we provide Lemma 5.1 to 
control the error incurred from both this interpolation and stopping after the $n$th iteration. To this effect, in order to include also the case of uncertain intensities, we shall consider the operator $T^{k}$ in (35) for some fixed $k \leq n$ instead of $T$. This will lead to a fixed contraction constant $1-p^{0}(k, 0)$ (see Lemma 4.2) also for the case of uncertain intensities and thus also for this case to the same upper bound in Lemma 5.1 below that goes to zero for $n \rightarrow \infty$.

For the case of unknown intensities we shall examine an approximation $J_{k}^{n}$ of $J^{n}$ defined recursively via

$$
J_{k}^{0}=0, \quad \text { and, for } h \leq n, \quad J_{k}^{h}=T^{k} J_{k}^{h-1},
$$

and restrict the arguments $v$ and $t$ to $(v, t) \in \mathcal{S}:=\left[-c, F_{k}\right] \times[0, S]$ with

$$
F_{k}:=\max \left\{F_{u, d} \mid u+d \leq k-1\right\} ;
$$

see (5). Notice in fact that, if at any time $v \geq F_{k}$, then superhedging is possible, and this leads to an optimal value $J^{*}=0$. Since for $v=F_{k}$ we are interested also in the optimal strategy, in what follows we shall thus require that $v \leq F_{k}$, i.e., $v \in\left[-c, F_{k}\right]$.

For the case of known intensities we shall use the same approximations as in (42), and all that follows holds in the same way also for this case by simply putting $k=n$.

Consider then some finite grid $G \subset \mathcal{S}$ containing the extremal points of $\mathcal{S}$ and denote by $\mathcal{D}\left(E^{k}\right)$ the Banach space of cadlag functions on $E^{k}$ endowed with the Skorokhod norm $\|\cdot\|_{E^{k}}$. Define the operator $T_{G}^{k}: \mathcal{D}\left(E^{k}\right) \rightarrow \mathcal{D}\left(E^{k}\right)$ via

$$
\left(T_{G}^{k} H\right)(v, u, d, t):= \begin{cases}(T H)(v, d, u, t) & \text { if }(v, t) \in G, u+d \leq k-1, \\ 0 & \text { if } u+d=k \text { or } v>F_{k}, \\ \text { cadlag interpolation } & \text { else. }\end{cases}
$$

Notice that, although for $v=F_{k}$ we have the possibility of superhedging, in our calculations we need to consider also this value in order to obtain the corresponding (superhedging) strategy. Due to our interpolation approximations, this strategy will, however, turn out to be only approximately superhedging.

Due to Corollary 4.3, we have

$$
T_{G}^{k} J^{*}= \begin{cases}J^{*}(v, d, u, t) & \text { if }(v, t) \in G, u+d \leq k-1, \\ 0 & \text { if } u+d=k \text { or } v>F_{k}, \\ \text { cadlag interpolation } & \text { else. }\end{cases}
$$

Due to the continuity of $J^{*}$, we have

$$
\epsilon(G):=\left\|J^{*}-T_{G}^{k} J^{*}\right\|_{E^{k-1}} \rightarrow 0
$$

for

$$
\sup _{(v, t) \in \mathcal{S}} \min _{\left(v^{\prime}, t^{\prime}\right) \in G}\left(\left|v-v^{\prime}\right|+\left|t-t^{\prime}\right|\right) \rightarrow 0 .
$$

We approximate the optimal value $J^{*}$ by the value $H_{k}^{n}$ defined recursively via

$$
H_{k}^{1}=J_{k}^{1}, \quad H_{k}^{n}=T_{G}^{k} H_{k}^{n-1} \quad \text { for } n \geq k .
$$

This value can be achieved by some strategy $\tilde{\xi}^{n}$ defined in analogy to (14)-(16) and, similarly, for the case of uncertain intensities. 
With relation (44), the next lemma provides a handle on the error.

LEMMA 5.1. We have

$$
\left\|J^{*}-H_{k}^{n}\right\|_{E^{k-1}} \leq \frac{1}{1-\kappa}\left(\kappa^{n}\left\|J_{k}^{1}\right\|_{E^{k-1}}+\epsilon(G)\right),
$$

where $\kappa$ is given by

(i) $\kappa=1-e^{-\lambda S}$ in the case of known intensities,

(ii) $\kappa=1-p^{0}(k, 0)$ in the case of uncertain intensities.

Proof. 1. It follows by an immediate extension to the case of cadlag interpolations of the proof of Lemma 3.1 (respectively, Lemma 4.2) that the operator $T_{G}^{k}: \mathcal{D}\left(E^{k}\right) \rightarrow$ $\mathcal{D}\left(E^{k}\right)$ is a contraction with contraction constant $\kappa$. Especially, $T_{G}^{k}$ has a fixed point $H_{k}^{*} \in \mathcal{D}\left(E^{k}\right)$. Noticing that $H_{k}^{1}(v, u, d, t)=p^{0}(u+d, t) l\left(F\left(x_{0} e^{a u-b d}\right)-v\right)$ and that, consequently,

$$
\begin{aligned}
\left\|H_{k}^{2}(v, u, d, t)-H_{k}^{1}(v, u, d, t)\right\|_{E^{k-1}} & =\left\|T_{G}^{k} H_{k}^{1}(v, u, d, t)-H_{k}^{1}(v, u, d, t)\right\|_{E^{k-1}} \\
& \leq\left|\int_{0}^{S-t}\left\{p^{+}(u, d, t, s)+p^{-}(u, d, t, s)\right\} d s\right|\left\|H_{k}^{1}\right\|_{E^{k-1}},
\end{aligned}
$$

one obtains

$$
\left\|H_{k}^{n}-H_{k}^{*}\right\|_{E^{k-1}} \leq \frac{\kappa^{n}}{1-\kappa}\left\|J_{k}^{1}\right\|_{E^{k-1}}
$$

2. We have

$$
\begin{aligned}
\left\|J^{*}-H_{k}^{*}\right\|_{E^{k-1}} & \leq\left\|J^{*}-T_{G}^{k} J^{*}\right\|_{E^{k-1}}+\left\|T_{G}^{k} J^{*}-T_{G}^{k} H_{k}^{*}\right\|_{E^{k-1}} \\
& \leq \epsilon(G)+\kappa\left\|J^{*}-H_{k}^{*}\right\|_{E^{k-1}}
\end{aligned}
$$

namely,

$$
\left\|J^{*}-H_{k}^{*}\right\|_{E^{k-1}} \leq \frac{1}{1-\kappa} \epsilon(G)
$$

3. Due to

$$
\left\|J^{*}-H_{k}^{n}\right\|_{E^{k-1}} \leq\left\|J^{*}-H_{k}^{*}\right\|_{E^{k-1}}+\left\|H_{k}^{n}-H_{k}^{*}\right\|_{E^{k-1}},
$$

the estimate (46) follows from 1 and 2.

By analogy to (26) and (41) we now have

$$
H_{k}^{n} \leq E\left[l\left(F\left(X_{S}\right)-\tilde{V}_{S}^{n}\right)\right] \leq H_{k}^{n}+E\left[l\left(F\left(X_{S}\right)+c\right), \tau_{k} \leq S\right],
$$

where $\tilde{V}_{t}^{n}$ is the wealth process associated with the strategy $\tilde{\xi}^{n}$.

Remark 4. The just-described algorithm hinges upon the specific form given to the operator $T^{k}$ in (35) for the case $u+d=k$. Variants are possible, and they imply slight variants also for the algorithm with advantages and disadvantages.

5.1. Example. Here we consider a simple example to illustrate the interpolation algorithm described above in this section. The example is as follows.

Consider the geometric Poisson price model (1) with $x_{0}=1$ and suppose that $a, b$ are such that $e^{a}=2, e^{-b}=\frac{1}{2}$. For the case of known intensities of the driving Poisson processes we let them be given by $\lambda^{+}=\lambda^{-}=1$, so that $\lambda=2$. For the case of unknown intensities we choose $\alpha_{0}^{+}=\alpha_{0}^{-}=\beta_{0}=1$ so that $\bar{\lambda}_{0}^{+}=\bar{\lambda}_{0}^{-}=1$. 
Take a claim of the form $F\left(X_{S}\right)=\left(X_{S}-1\right)^{+}$, a time horizon of $S=2$, and put $c=0.5$. The superhedge price for $F$ is (see the comment after (5)) $F_{u, d} \equiv x_{0}=1$. Let the loss function be of the form $l(z)=[\max (z, 0)]^{p}$ for $p=2$. The optimization criterion is then

$$
J_{0}^{*}:=\inf _{\xi \in \mathcal{A}_{V_{0}}} E\left\{\left[\max \left(\left(x_{0} e^{a u-b d}-1\right)^{+}-V_{S}, 0\right)\right]^{2}\right\}
$$

and it is of the type of shortfall risk minimization. The interval of admissible values for $\xi$ on $\left[0, \hat{\tau}_{1}\right]$ is given by (see (9))

$$
I_{v, u, d}=\left[-\frac{c+v}{2^{u-d}}, 2 \frac{c+v}{2^{u-d}}\right]
$$

Since we have $F_{u, d} \equiv x_{0}=1$, also for $F_{k}$ in (43) one has $F_{k} \equiv 1$. This implies that, for the given data, $(v, t) \in \mathcal{S}:=\left[-\frac{1}{2}, 1\right] \times[0,2]$. Consider then the following grid that, for the purpose of illustrating the procedure in the simplest possible way, is chosen to be very coarse. More precisely, $G \subset \mathcal{S}$ is obtained as follows: partition the time interval $[0,2]$ into $L=2$ subintervals $T_{0}:=\left[t_{0}=0, t_{1}=1\right], T_{1}:=\left[t_{1}=1, t_{2}=2\right]$ and the wealth-value interval $\left[-\frac{1}{2}, 1\right]$ into $M=3$ subintervals $V_{0}:=\left[v_{0}=-\frac{1}{2}, v_{1}=\right.$ $0], V_{1}:=\left[v_{1}=0, v_{2}=\frac{1}{4}\right], V_{2}:=\left[v_{2}=\frac{1}{4}, v_{3}=1\right]$. The reason why we consider $v_{2}=\frac{1}{4}$ and not, say, $v_{2}=\frac{1}{2}$ is that at $v=\frac{1}{2}$ the optimal values are already equal to zero. Even though we are still far from the superhedge value of $v=1$, this happens because we restrict ourselves to the event that no more than $k$ jumps can occur.

5.1.1. Known intensities. We shall compute the values for $H_{k}^{n}$ according to (45) (as well as (42)) for tuples $\left(v_{j}, u, d, t_{i}\right)$ with $i \in\{0,1\}, j \in\{0,1,2\}$, and $u+d \leq$ $k-1$. In fact, this last restriction for $u$ and $d$ and the sufficiency of considering only values of $v \leq 1$ come from the definition of the operator $T_{G}^{k}$. On the other hand, since we perform a cadlag interpolation, we do not need to consider the upper end point $t_{i}=2$ of the time interval; i.e., it suffices to have $i \in\{0,1\}$.

First we recall from (42) that, for our example,

$$
J_{k}^{1}\left(v_{j}, u, d, t_{i}\right)=e^{-2\left(2-t_{i}\right)}\left[\max \left\{\left(2^{u-d}-1\right)^{+}-v_{j}, 0\right\}\right]^{2}, \quad i \in\{0,1\}, j \in\{0,1,2\},
$$

and notice that (see by analogy the motivation for (21))

$$
J_{k}^{1}\left(v_{j}, u, d, t_{i}+s\right)=e^{2 s} J_{k}^{1}\left(v_{j}, u, d, t_{i}\right) .
$$

The recursions (45) now become, always for the case of our example and recalling 
that we consider cadlag interpolations,

$$
\begin{aligned}
& H_{k}^{n}\left(v_{j}, u, d, t_{i}\right)=\left(T_{G}^{k} H_{k}^{n-1}\right)\left(v_{j}, u, d, t_{i}\right) \\
& =J_{k}^{1}\left(v_{j}, u, d, t_{i}\right)+\int_{0}^{S-t_{i}} e^{-2 s} \\
& \min _{\zeta \in I_{v_{j}, u, d}}\left\{\begin{array}{l}
\sum_{l=0}^{L-1} \sum_{m=0}^{M-1} 1_{\left\{T_{l}\right\}}\left(t_{i}+s\right) 1_{\left\{V_{m}\right\}}\left(v_{j}+\zeta x_{0} e^{a u-b d}\left(e^{a}-1\right)\right) \\
H_{k}^{n-1}\left(v_{m}, u+1, d, t_{l}\right) \\
+\sum_{l=0}^{L-1} \sum_{m=0}^{M-1} 1_{\left\{T_{l}\right\}}\left(t_{i}+s\right) 1_{\left\{V_{m}\right\}}\left(v_{j}+\zeta x_{0} e^{a u-b d}\left(e^{-b}-1\right)\right) \\
H_{k}^{n-1}\left(v_{m}, u, d+1, t_{l}\right)
\end{array}\right\} d s \\
& =J_{k}^{1}\left(v_{j}, u, d, t_{i}\right)+\sum_{l=0}^{L-1} 1_{\left\{t_{i} \leq t_{l}\right\}} \gamma_{i, l} \\
& \min _{\zeta \in I_{v_{j}, u, d}}\left\{\begin{array}{l}
M-1 \\
\sum_{m=0}^{M-1} 1_{\left\{V_{m}\right\}}\left(v_{j}+\zeta x_{0} e^{a u-b d}\left(e^{a}-1\right)\right) H_{k}^{n-1}\left(v_{m}, u+1, d, t_{l}\right) \\
+\sum_{m=0}^{M-1} 1_{\left\{V_{m}\right\}}\left(v_{j}+\zeta x_{0} e^{a u-b d}\left(e^{-b}-1\right)\right) H_{k}^{n-1}\left(v_{m}, u, d+1, t_{l}\right)
\end{array}\right\},
\end{aligned}
$$

where we have used the fact that

$$
\int_{0}^{S-t_{i}} e^{-2 s} 1_{\left[t_{l}, t_{l+1}\right)}\left(t_{i}+s\right) d s=\sum_{l=0}^{L-1} 1_{\left\{t_{i} \leq t_{l}\right\}} \frac{e^{2 t_{i}}}{2}\left[e^{-2 t_{l}}-e^{-2 t_{l+1}}\right]
$$

so that $\gamma_{i, l}:=\frac{e^{2 t_{i}}}{2}\left[e^{-2 t_{l}}-e^{-2 t_{l+1}}\right]$, in particular $\gamma_{0,0}=\frac{1-e^{-2}}{2} \gamma_{0,1}=e^{-2} \gamma_{0,0}$. Since $J_{k}^{1}$ satisfies property (50), we have that for $n=2$ the above recursions (51) simplify to become

$$
\begin{aligned}
H_{k}^{2}\left(v_{j}, u, d, t_{i}\right)=J_{k}^{1}\left(v_{j}, u, d, t_{i}\right) \\
+\left(S-t_{i}\right) \cdot \min _{\zeta \in I_{v_{j}, u, d}}\left\{\begin{array}{l}
\sum_{m=0}^{M-1} 1_{\left\{V_{m}\right\}}\left(v_{j}+\zeta x_{0} e^{a u-b d}\left(e^{a}-1\right)\right) J_{k}^{1}\left(v_{m}, u+1, d, t_{i}\right) \\
+\sum_{m=0}^{M-1} 1_{\left\{V_{m}\right\}}\left(v_{j}+\zeta x_{0} e^{a u-b d}\left(e^{-b}-1\right)\right) J_{k}^{1}\left(v_{m}, u, d+1, t_{i}\right)
\end{array}\right\} .
\end{aligned}
$$

Always in order to illustrate the procedure in the simplest possible way, we shall now consider the case of $k=n=3$ with the objective of computing the values for $H_{3}^{3}(0,0,0,0)$ and $H_{3}^{3}\left(\frac{1}{4}, 0,0,0\right)$ as well as the corresponding minimizing strategy $\xi_{s}^{3}$ for $s \in[0,2]$.

For this purpose we first compute $J_{3}^{1}\left(v_{j}, u, d, t_{i}\right)$ for $v_{j} \in\left\{-\frac{1}{2}, 0, \frac{1}{4}, 1\right\}, t_{i} \in\{0,1\}$, and $u+d \leq 2$. This can be easily done on the basis of (49) noticing that these values are zero for all tuples $\left(v_{j}, u, d, t_{i}\right)$ with $u \leq d$ and $v_{j} \geq 0$ and this is, thanks to (50), independently of $t_{i}$. 
One then proceeds to compute $H_{3}^{2}\left(v_{j}, u, d, t_{i}\right)$ for $v_{j} \in\left\{-\frac{1}{2}, 0, \frac{1}{4}, 1\right\}, t_{i} \in\{0,1\}$, and $u+d=1$. Notice that the computation of these values leads then also to the minimizing strategy for $s \in\left(\hat{\tau}_{1}, \hat{\tau}_{2}\right]$. Since by (48) we have $I_{-\frac{1}{2}, u, d}=\{0\}$, no minimization is required for the computation of $H_{3}^{2}\left(-\frac{1}{2}, u, d, t_{i}\right)$, and so, for this case of $v_{j}=-\frac{1}{2}$, the minimizing strategy is $\xi_{s} \equiv 0$ when $s \in\left(\hat{\tau}_{1}, \hat{\tau}_{2}\right]$. From (52) we now have

$$
\begin{aligned}
H_{3}^{2}\left(-\frac{1}{2}, 1,0,0\right) & =J_{3}^{1}\left(-\frac{1}{2}, 1,0,0\right)+2\left\{J_{3}^{1}\left(-\frac{1}{2}, 2,0,0\right)+J_{3}^{1}\left(-\frac{1}{2}, 1,1,0\right)\right\} \\
& =\frac{109}{4} e^{-4} .
\end{aligned}
$$

With similar calculations one obtains $H_{3}^{2}\left(-\frac{1}{2}, 0,1,0\right)=\frac{5}{4} e^{-4}$, and, from here, by (50) $H_{3}^{2}\left(-\frac{1}{2}, 1,0,1\right)=\frac{59}{4} e^{-2}$ and $H_{3}^{2}\left(-\frac{1}{2}, 0,1,1\right)=\frac{3}{4} e^{-2}$.

Moving on to $v_{j}=0$, we have that $I_{0, u, d}=\left[-\frac{c}{2^{u-d}}, 2 \frac{c}{2^{u-d}}\right] \neq\{0\}$ so that this time one has to perform also the minimization according to (52). For this purpose notice that the partition $\left\{V_{m}\right\}_{m=1}^{M}$ of the wealth-value interval induces a partition of $I_{0, u, d}$ characterized by the fact that the right-hand side in (52) remains constant for all values of $\zeta$ in a same subinterval of the partition. We thus have

$$
\begin{aligned}
H_{3}^{2}(0,1,0,0)= & J_{3}^{1}(0,1,0,0) \\
+ & 2 \min \left\{1_{\left\{\zeta=-\frac{1}{4}\right\}}\left[J_{3}^{1}\left(-\frac{1}{2}, 2,0,0\right)+J_{3}^{1}\left(\frac{1}{4}, 1,1,0\right)\right],\right. \\
& 1_{\left\{\zeta \in\left(-\frac{1}{4}, 0\right)\right\}}\left[J_{3}^{1}\left(-\frac{1}{2}, 2,0,0\right)+J_{3}^{1}(0,1,1,0)\right], \\
& 1_{\{\zeta=0\}}\left[J_{3}^{1}(0,2,0,0)+J_{3}^{1}(0,1,1,0)\right], \\
& 1_{\left\{\zeta \in\left(0, \frac{1}{8}\right)\right\}}\left[J_{3}^{1}(0,2,0,0)+J_{3}^{1}\left(-\frac{1}{2}, 1,1,0\right)\right], \\
& 1_{\left\{\zeta \in\left(\frac{1}{8}, \frac{1}{8}\right)\right\}}\left[J_{3}^{1}\left(\frac{1}{4}, 2,0,0\right)+J_{3}^{1}\left(-\frac{1}{2}, 1,1,0\right)\right], \\
& \left.1_{\left\{\zeta=\frac{1}{2}\right\}}\left[J_{3}^{1}(1,2,0,0)+J_{3}^{1}\left(-\frac{1}{2}, 1,1,0\right)\right]\right\} \\
=e^{-4}+ & 2 \min \left\{\frac{49}{4} e^{-4}, \frac{49}{4} e^{-4}, 9 e^{-4}, \frac{37}{4} e^{-4}, \frac{125}{16} e^{-4}, \frac{17}{4} e^{-4}\right\}=\frac{19}{2} e^{-4},
\end{aligned}
$$

where the min is achieved for $\zeta=\frac{1}{2}$ so that, for this case of $v_{j}=0$, the minimizing strategy is $\xi_{s}^{3}=\frac{1}{2}$ when $s \in\left(\hat{\tau}_{1}, \hat{\tau}_{2}\right] \cap[0,1]$. Analogously, one obtains $H_{3}^{2}(0,1,0,1)=$ $\frac{21}{4} e^{-2}$ where, thanks to $(50)$, the minimizing value of $\zeta$ is the same as before so that we have again $\xi_{s}^{3}=\frac{1}{2}$ for $v_{j}=0$ also when $s \in\left(\hat{\tau}_{1}, \hat{\tau}_{2}\right] \cap[(1,2]$. By similar calculations one then obtains

$$
\begin{array}{ll}
H_{3}^{2}(0,0,1,0)=H_{3}^{2}(0,0,1,1)=0 & \text { with minimizing } \zeta=0, \\
H_{3}^{2}\left(\frac{1}{4}, 1,0,0\right)=\frac{145}{16} e^{-4} & \text { with minimizing } \zeta=\frac{3}{8}, \\
H_{3}^{2}\left(\frac{1}{4}, 1,0,1\right)=\frac{77}{16} e^{-2} & \text { with minimizing } \zeta=\frac{3}{8}, \\
H_{3}^{2}\left(\frac{1}{4}, 0,1,0\right)=H_{3}^{2}\left(\frac{1}{4}, 0,1,1\right)=0 & \text { with minimizing } \zeta \text { any } \zeta \in\left[-\frac{1}{2}, 1\right], \\
H_{3}^{2}(1,1,0,0)=8 e^{-4} & \text { with minimizing } \zeta=0, \\
H_{3}^{2}(1,1,0,1)=4 e^{-2} & \text { with minimizing } \zeta=0 .
\end{array}
$$


At this point one can finally compute

$$
\begin{aligned}
& H_{3}^{3}(0,0,0,0)=J_{3}^{1}(0,0,0,0) \\
& +\sum_{l=0}^{1} \gamma_{0, l} \min \left\{1_{\left\{\zeta=-\frac{1}{2}\right\}}\left[H_{3}^{2}\left(-\frac{1}{2}, 1,0, t_{l}\right)+H_{3}^{2}\left(\frac{1}{4}, 0,1, t_{l}\right)\right],\right. \\
& 1_{\left\{\zeta \in\left(-\frac{1}{2}, 0\right)\right\}}\left[H_{3}^{2}\left(-\frac{1}{2}, 1,0, t_{l}\right)+H_{3}^{2}\left(0,0,1, t_{l}\right)\right], \\
& 1_{\{\zeta=0\}}\left[H_{3}^{2}\left(0,1,0, t_{l}\right)+H_{3}^{2}\left(0,0,1, t_{l}\right)\right], \\
& 1_{\left\{\zeta \in\left(0, \frac{1}{4}\right)\right\}}\left[H_{3}^{2}\left(0,1,0, t_{l}\right)+H_{3}^{2}\left(-\frac{1}{2}, 0,1, t_{l}\right)\right], \\
& 1_{\left\{\zeta \in\left[\frac{1}{4}, 1\right)\right\}}\left[H_{3}^{2}\left(\frac{1}{4}, 1,0, t_{l}\right)+H_{3}^{2}\left(-\frac{1}{2}, 0,1, t_{l}\right)\right], \\
& \left.1_{\{\zeta=1\}}\left[H_{3}^{2}\left(1,1,0, t_{l}\right)+H_{3}^{2}\left(-\frac{1}{2}, 0,1, t_{l}\right)\right]\right\} \\
& =7 e^{-4}\left(1-e^{-2}\right),
\end{aligned}
$$

where the min is achieved for $\zeta=1$ both when $t_{l}=0$ and when $t_{l}=1$ so that, for $v_{j}=0$, the minimizing strategy is $\xi_{s}^{3}=1$ when $s \in\left[0, \hat{\tau}_{1}\right] \cap[0,2]$. Peforming analogous calculations one obtains that $H_{3}^{3}\left(\frac{1}{4}, 0,0,0\right)=\frac{221}{32} e^{-4}\left(1-e^{-2}\right)$, which is slightly less than the value for $H_{3}^{3}(0,0,0,0)$, and the min here is achieved for any $\zeta \in\left[0, \frac{1}{2}\right]$ when $t_{l}=0$ and for $\zeta=\frac{3}{4}$ when $t_{l}=1$. This implies that, for $v_{j}=\frac{1}{4}$, the minimizing strategy is $\xi_{s}^{3} \in\left[0, \frac{1}{2}\right]$ when $s \in\left(0, \hat{\tau}_{1}\right] \cap[0,1]$ and $\xi_{s}^{3}=\frac{3}{4}$ when $s \in\left(0, \hat{\tau}_{1}\right] \cap(1,2]$.

Summarizing, we have obtained for the (approximating) minimizing strategy the following expression where, due to the right continuous interpolation, the strategy is the same for all values $v$ of the wealth belonging to a same subinterval of the partition $\left\{V_{m}\right\}$ and given by the value computed in its lower end point. We have in fact

$$
\xi_{s}^{3}= \begin{cases}1 & \text { if } s \in\left[0, \hat{\tau}_{1}\right] \cap[0,2] \text { and } V_{0}=0, \\ \text { any } \zeta \in\left[0, \frac{1}{2}\right] & \text { if } s \in\left[0, \hat{\tau}_{1}\right] \cap[0,1] \text { and } V_{0}=\frac{1}{4}, \\ \zeta=\frac{3}{4} & \text { if } s \in\left[0, \hat{\tau}_{1}\right] \cap(1,2] \text { and } V_{0}=\frac{1}{4}, \\ \zeta=\frac{3}{8} & \text { if } s \in\left(\hat{\tau}_{1}, \hat{\tau}_{2}\right] \cap[0,2], u=1, d=0, V_{\hat{\tau}_{1}} \in\left[\frac{1}{4}, 1\right), \\ \zeta=0 & \text { if } s \in\left(\hat{\tau}_{1}, \hat{\tau}_{2}\right] \cap[0,2], u=1, d=0, V_{\hat{\tau}_{1}} \geq 1, \\ 0 & \text { if } s \in\left(\hat{\tau}_{1}, \hat{\tau}_{2}\right] \cap[0,2], u=0, d=1, V_{\hat{\tau}_{1}}<0, \\ 0 & \text { if } s \in\left(\hat{\tau}_{1}, \hat{\tau}_{2}\right] \cap[0,2], u=0, d=1, V_{\hat{\tau}_{1}} \in\left[0, \frac{1}{4}\right), \\ \text { any } \zeta \in\left[-\frac{1}{2}, 1\right] & \text { if } s \in\left(\hat{\tau}_{1}, \hat{\tau}_{2}\right] \cap[0,2], u=0, d=1, V_{\hat{\tau}_{1}}=\frac{1}{4}, \\ 0 & \text { if } s>\hat{\tau}_{2} .\end{cases}
$$

5.1.2. Uncertain intensities. We choose $\alpha_{0}^{+}=\alpha_{0}^{-}=\beta_{0}=1$ so that $\bar{\lambda}_{0}^{+}=$ $\bar{\lambda}_{0}^{-}=1$. We start from the expression for $J_{k}^{1}$ that is given here by (see (42))

$J_{k}^{1}\left(v_{j}, u, d, t_{i}\right)$ 


$$
=\left(\frac{t_{i}+1}{3}\right)^{u+d+2}\left[\max \left\{\left(2^{u-d}-1\right)^{+}-v_{j}, 0\right\}\right]^{2}, i \in\{0,1\}, j \in\{0,1,2\},
$$

for which, analogously to (50), we have

$$
J_{k}^{1}\left(v_{j}, u, d, t+s\right)=\left(\frac{t+s+1}{t+1}\right)^{u+d+2} J_{k}^{1}\left(v_{j}, u, d, t\right) .
$$

On the other hand, the recursions (45) become

$$
\begin{aligned}
H_{k}^{n}\left(v_{j}, u, d, t_{i}\right)= & J_{k}^{1}\left(v_{j}, u, d, t_{i}\right) \\
& +\int_{0}^{S-t_{i}} \min _{\zeta \in I_{v_{j}, u, d}}\left\{\sum_{l, m=0}^{L-1} 1_{\left\{T_{l}\right\}}\left(t_{i}+s\right)\left(\frac{1+t_{i}}{1+t_{l}}\right)^{1+u+d} \frac{1+u}{1+t_{l}}\right. \\
& \cdot 1_{\left\{V_{m}\right\}}\left(v_{j}+\zeta x_{0} e^{a u-b d}\left(e^{a}-1\right)\right) H_{k}^{n-1}\left(v_{m}, u+1, d, t_{l}\right)
\end{aligned}
$$

having put

$$
\left\{\begin{array}{l}
\gamma_{i, l}^{u}=\left(t_{l+1}-t_{l}\right)\left(\frac{1+t_{i}}{1+t_{l}}\right)^{1+u+d} \frac{1+u}{1+t_{l}}, \\
\gamma_{i, l}^{d}=\left(t_{l+1}-t_{l}\right)\left(\frac{1+t_{i}}{1+t_{l}}\right)^{1+u+d} \frac{1+d}{1+t_{l}} .
\end{array}\right.
$$

Due to (54) the relations (55) simplify slightly for $n=2$ but not anymore as much as in $(52)$.

At this point the procedure parallels mostly the one described in the previous subsection for known values of the intensities and the calculations are similar.

Note added in proof. Improvements and extensions to the contents of section 5 can be found in [19].

\section{REFERENCES}

[1] A. Almudevar, A dynamic programming algorithm for the optimal control of piecewise deterministic Markov processes, SIAM J. Control Optim., 40 (2001), pp. 525-539. 
[2] J. CVITANIĆ, Minimizing expected loss of hedging in incomplete and constrained markets, SIAM J. Control Optim., 38 (2000), pp. 1050-1066.

[3] J. Cvitanić and I. Karatzas, Hedging and portfolio optimization under transaction costs: A martingale approach, Math. Finance, 6 (1996), pp. 133-165.

[4] J. CVitanić And I. Karatzas, On dynamic measures of risk, Finance Stoch., 4 (1999), pp. 451482.

[5] M. H. A Davis, Piecewise-deterministic Markov processes: A general class of non-diffusion stochastic models, J. R. Stat. Soc. Ser. B Stat. Methodol., 46 (1984), pp. 353-388.

[6] M. A. H. Dempster And J. J. Ye, Necessary and sufficient optimality conditions for control of piecewise deterministic Markov processes, Stochastics Stochastics Rep., 40 (1992), pp. 125145.

[7] N. El KARoui AND M.-C. Quenez, Dynamic programming and pricing of contingent claims in an incomplete market, SIAM J. Control Optim., 33 (1995), pp. 29-66.

[8] H. Föllmer and P. Leukert, Efficient hedging: Cost versus shortfall risk, Finance Stoch., 4 (2000), pp. 117-146.

[9] H. Föllmer And D. Sondermann, Hedging of non-redundant contingemt claims, in Contributions to Mathematical Economics in Honor of Géerard Debreu, W. Hildenbrand and A. Mas-Colell, eds., North-Holland, Amsterdam, 1986, pp. 205-223.

[10] R. FRey AND W. J. RungGaldieR, Risk-minimizing hedging strategies under restricted information: The case of stochastic volatility models observed only at discrete random times, Math. Methods Oper. Res., 50 (1999), pp. 339-350.

[11] A. A. Gushchin And E. Mordecki, Bounds on option prices for semimartingale market models, Proc. Steklov Inst. Math., 237 (2002), pp. 73-113.

[12] O. Hernández-Lerma, Adaptive Markov Control Processes, Springer-Verlag, Berlin, 1989.

[13] I. Karatzas and S. E. Shreve, Methods of Mathematical Finance, Springer-Verlag, New York, 1998.

[14] I. Karatzas and X. ZhaO, Bayesian adaptive portfolio optimization, in Handbook of Mathematical Finance, Cambridge University Press, Cambridge, UK, 2001, pp. 632-670.

[15] M. KIRCH, Efficient Hedging in Incomplete Markets under Model Uncertainty, Ph.D. thesis, Humboldt University, Berlin, Germany, 2002.

[16] R. Korn, Optimal Portfolios. Stochastic Models for Optimal Investment and Risk Management in Continuous Time, World Scientific, Singapore, 1997.

[17] D. Kramkov, Optional decomposition of supermartingales and hedging contingent claims in incomplete security markets, Probab. Theory Related Fields, 105 (1996), pp. 459-479.

[18] P. LAKNer, Utility maximization with partial information, Stochastic Process. Appl., 56 (1995), pp. 247-273.

[19] W. J. RungGaldier And S. Di Emidio, Computing efficient hedging strategies in discontinuous market models, in Proceedings of International Conference "Stochastic Finance 2004," Lisbon, Portugal, 2004.

[20] R. Rishel, Optimal portfolio management with partial observations and power utility function, in Stochastic Analysis, Control, Optimization and Applications, Volume in Honor of W. H. Fleming, W. M. McEneany, G. Yin, and Q. Zhang, eds., Birkhäuser, Boston, 1999, pp. 605619.

[21] W. Schachermayer, Optimal investment in incomplete financial markets, in Mathematical Finance-Bachelier Congress 2000, H. Geman, D. Madan, S. Pliska, and T. Vorst, eds., Springer-Verlag, Berlin, 2002, pp. 427-462.

[22] M. SchweIzer, Mean variance hedging for general claims, Ann. Appl. Probab., 2 (1992), pp. 171-179.

[23] M. SchweIzer, Risk minimizing hedging strategies under restricted information, Math. Finance, 4 (1994), pp. 327-342.

[24] M. SchweIzer, A guided tour through quadratic hedging approaches, in Option Pricing, Interest Rates and Risk Management, E. Jouini, J. Cvitanić, and M. Musiela, eds., Cambridge University Press, Cambridge, UK, 2000, pp. 538-574.

[25] D. Vermes, Optimal control of piecewise deterministic Markov processes, Stochastics, 14 (1985), pp. 165-208.

[26] G. ZohaR, A generalized Cameron-Martin formula with applications to partially observed dynamic portfolio optimization, Math. Finance, 11 (2001), pp. 475-494. 